with HIV. However, granulomatous reactions, lymphocytic infiltration, and elevated levels of CSF proinflammatory cytokines have been described. ${ }^{1,2}$ The degree of inflammation in patients who are receiving HAART is unknown but is probably higher than in patients who are not receiving HAART. Our trial was designed to aid decision making at the point of diagnosis. A total of $40 \%$ of the patients in our trial were receiving HAART at trial entry. However, we found no benefit of dexamethasone, even in this predefined subgroup analysis. As Miller notes, IRIS occurred infrequently in both trial groups by 6 months (in 6 of 226 patients in the placebo group and in 7 of 224 patients in the dexamethasone group). The low rate of IRIS is interesting; we speculate that it was due to the use of higher doses of antifungal agents (particularly fluconazole) than were typically used in previous cohorts.

Brandt questions the appropriateness of the regimen chosen. We chose the lowest dexamethasone dose used in the trial involving patients with tuberculous meningitis reported on by Thwaites et al. because in that trial patients who received active drug had fewer serious adverse events than patients who received placebo. ${ }^{3}$ Among the patients in the trial by Thwaites et al., 18\% were infected with HIV and most had profound immunosuppression; no evidence of harm was seen.
Survival in relation to the Glasgow Coma Scale score at admission is provided in Table 2 of our article, and survival in relation to raised intracranial pressure is described in Section 10 of the Supplementary Appendix (available with the full text of our article at NEJM.org). We think that allcause mortality is the most clinically meaningful end point and that our conclusions are robust.

Justin Beardsley, M.B., Ch.B.

Marcel Wolbers, Ph.D.

Jeremy N. Day, Ph.D.

Oxford University Clinical Research Unit

Ho Chi Minh City, Vietnam

jday@oucru.org

\section{for the CryptoDex Investigators}

Since publication of their article, the authors report no further potential conflict of interest.

1. Jarvis JN, Meintjes G, Bicanic T, et al. Cerebrospinal fluid cytokine profiles predict risk of early mortality and immune reconstitution inflammatory syndrome in HIV-associated cryptococcal meningitis. PLoS Pathog 2015;11(4):e1004754.

2. Klock C, Cerski M, Goldani LZ. Histopathological aspects of neurocryptococcosis in HIV-infected patients: autopsy report of 45 patients. Int J Surg Pathol 2009;17:444-8.

3. Day J, Imran D, Ganiem AR, et al. CryptoDex: a randomised, double-blind, placebo-controlled phase III trial of adjunctive dexamethasone in HIV-infected adults with cryptococcal meningitis: study protocol for a randomised control trial. Trials 2014; 15:441.

DOI: 10.1056/NEJMc1605205

\title{
Amoxicillin for Severe Acute Malnutrition in Children
}

TO THE EDITOR: Isanaka and colleagues (Feb. 4 issue $)^{1}$ report the results of a double-blind, placebo-controlled trial that showed no benefit from the routine use of amoxicillin in a cohort of children with uncomplicated severe malnutrition in Niger. The authors conclude that amoxicillin may not have a place in the empirical management of uncomplicated severe malnutrition in developing countries. We strongly support the view that antibacterial chemotherapy is not a magic bullet, and we wish to highlight the potential unintended consequences of the mass use of antibacterial agents in this context, including the potential for drug resistance. With the World Health Organization giving a central role to amoxicillin in its essential medicines list, ${ }^{2}$ and recent data con- firming that the use of antimicrobial agents in childhood is associated with long-lasting shifts in the intestinal microbiome, ${ }^{3}$ we call for a shift of emphasis away from the mass administration of antibacterial agents. Instead, ensuring that essential antibacterial agents for the treatment of clinical infections are accessible for all, while sustaining their effectiveness, must remain central to our global approach to managing infections - now and for generations to come. ${ }^{4}$

Timothy M. Rawson, M.B., B.S., D.T.M.\&H.

Luke S. Moore, D.T.M.\&H., M.P.H.

Alison H. Holmes, M.D., M.P.H.

Imperial College London

London, United Kingdom

tmr07@ic.ac.uk 
Dr. Moore reports receiving consulting fees from bioMérieux and DNA electronics; and Dr. Holmes, consulting fees from bioMérieux. No other potential conflict of interest relevant to this letter was reported.

1. Isanaka S, Langendorf C, Berthé F, et al. Routine amoxicillin for uncomplicated severe acute malnutrition in children. N Engl J Med 2016;374:444-53.

2. Vitoria M. WHO model list of essential medicines for children. Geneva: World Health Organization, August 2015.

3. Korpela K, Salonen A, Virta LJ, et al. Intestinal microbiome is related to lifetime antibiotic use in Finnish pre-school children. Nat Commun 2016;7:10410.

4. Årdal C, Outterson K, Hoffman SJ, et al. International cooperation to improve access to and sustain effectiveness of antimicrobials. Lancet 2016;387:296-307.

DOI: 10.1056/NEJMc1605388

TO THE EDITOR: Isanaka et al. conducted a trial involving only a subgroup of children who meet the case definition of severe acute malnutrition, excluding those with kwashiorkor., ${ }^{1,2}$ As two manifestations of severe acute malnutrition, marasmus and kwashiorkor are typically treated with the same therapeutic regimen. ${ }^{3}$ The availability of physicians and the high rate of transfers to inpatient care do not reflect operational conditions, limiting generalizability. The censoring of outcomes at the time of recommendation for hospitalization, even though half the children with such a recommendation were not actually admitted, skews the results toward a failure of therapy. We disagree that the routine use of antibiotic agents is unnecessary in children with marasmus. Finding no significant benefit is quite different from asserting that there is no benefit, especially when the lower boundary of the $95 \%$ confidence interval for a benefit with amoxicillin is 0.99 . The direction and magnitude of their results are fully consistent with a previous trial conducted in Malawi, ${ }^{4}$ which also showed a trend toward higher rates of recovery among children with marasmus who were treated with amoxicillin. Combining the results of these trials shows that 946 of 1396 children $(67.8 \%)$ who were treated with amoxicillin recovered, as compared with 897 of $1395(64.3 \%)$ who received placebo (risk ratio, 1.08; 95\% confidence interval [CI], 1.00 to 1.37; $\mathrm{P}=0.05$ by Mantel-Haenszel analysis).

Indi Trehan, M.D., M.P.H.

Kenneth B. Schechtman, Ph.D.

Mark J. Manary, M.D.

Washington University in St. Louis

St. Louis, MO

indi@alum.berkeley.edu
No potential conflict of interest relevant to this letter was reported.

1. WHO child growth standards and the identification of severe acute malnutrition in infants and children. Geneva: World Health Organization, United Nations Children's Fund, 2009.

2. Frison S, Checchi F, Kerac M. Omitting edema measurement: how much acute malnutrition are we missing? Am J Clin Nutr 2015;102:1176-81.

3. Updates on the management of severe acute malnutrition in infants and children. Geneva: World Health Organization, 2013. 4. Trehan I, Goldbach HS, LaGrone LN, et al. Antibiotics as part of the management of severe acute malnutrition. $\mathrm{N}$ Engl J Med 2013;368:425-35.

DOI: 10.1056/NEJMc1605388

THE AUTHORS REPLY: Rawson et al. highlight the importance of antibiotic stewardship, and we agree that the prudent use of existing antibiotics is essential to sustain their long-term effectiveness. Although Trehan and colleagues conducted a trial in Malawi ${ }^{1}$ that was similar to ours, we disagree on all three points. First, although our trial included only children with marasmus, we do not agree that this represents only a subgroup of children who meet the case definition of severe acute malnutrition. The comprehensive analysis of 852 nutritional surveys, ${ }^{2}$ also cited by Trehan et al., confirms that the overall burden of severe acute malnutrition differs little with the exclusion of edema and substantiates the conclusion that marasmic severe acute malnutrition represents the preponderance of cases worldwide. In Niger, $1 \%$ of the children who were screened for eligibility in our trial were excluded owing to kwashiorkor (Fig. 1 of our article), which similarly suggests a minimal consequence of the exclusion on the overall generalizability of the trial findings.

Second, we disagree that the discontinuation of follow-up at the time of transfer to inpatient care represents a "censoring of outcomes." In our trial, the secondary outcome under evaluation was first transfer to inpatient care. Discontinuation of follow-up at this moment is therefore not informative censoring that produces bias; rather, it is a result of achieving an outcome of clinical and programmatic interest as intended in our protocol. Third, we appreciate the importance of considering effect size, boundaries of confidence intervals, and significance levels, ${ }^{3}$ but we maintain the interpretation of our findings that a benefit of routine amoxicillin in terms of nutritional recovery was not confirmed in our trial. The pooled analysis presented by Trehan et al. 
confirms that there is no significant benefit $(\mathrm{P}<0.05)$ of routine amoxicillin with regard to recovery during treatment (risk ratio, 1.08; 95\% $\mathrm{CI}, 1.00$ to $1.37 ; \mathrm{P}=0.05$ ). We do, however, agree on the challenges in generalizing research findings to operational settings. This limitation was recognized in the article, and we are currently supporting efforts to confirm the research findings in the context of a routine nutrition treatment program with Médecins sans Frontières in Niger.

Sheila Isanaka, Sc.D.

Epicentre

Paris, France

Eric Adehossi, M.D.

National Hospital

Niamey, Niger
Rebecca F. Grais, Ph.D.

Epicentre

Paris, France

rebecca.grais@epicentre.msf.org

Since publication of their article, the authors report no further potential conflict of interest.

1. Trehan I, Goldbach HS, LaGrone LN, et al. Antibiotics as part of the management of severe acute malnutrition. N Engl J Med 2013;368:425-35.

2. Frison S, Checchi F, Kerac M. Omitting edema measurement: how much acute malnutrition are we missing? Am J Clin Nutr 2015;102:1176-81.

3. Chavalarias D, Wallach JD, Li AH, Ioannidis JP. Evolution of reporting $\mathrm{P}$ values in the biomedical literature, 1990-2015. JAMA 2016;315:1141-8.

DOI: 10.1056/NEJMc1605388

\section{Eliminating Pediatric HIV-1 Infection}

TO THE EDITOR: We agree with Luzuriaga and Mofenson (Feb. 25 issue) $^{1}$ that it is possible to contemplate the elimination of mother-to-child transmission of human immunodeficiency virus type 1 (HIV-1) in the United States and in resource-limited areas. To that end, the framework at the Centers for Disease Control and Prevention (CDC) for the elimination of mother-to-child transmission of HIV-1 in the United States uses two targets: an incidence of less than 1 case per 100,000 live births and a mother-to-child transmission rate of less than $1 \%{ }^{2}$ Regarding the rate

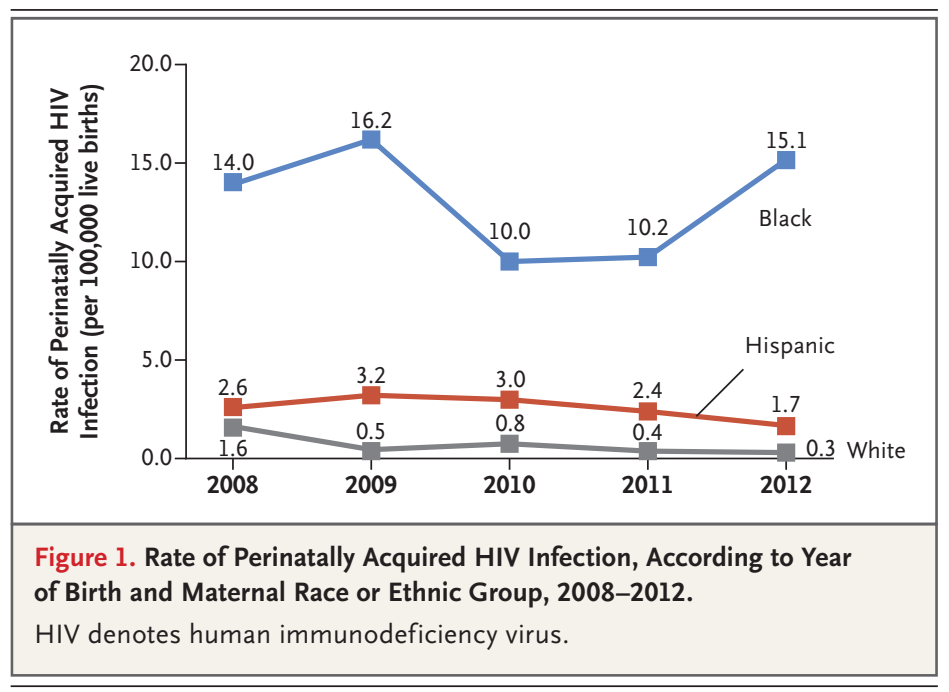

of less than $1 \%$ that is attributed to the United States in the article, the authors cite data from the United Kingdom and Ireland. ${ }^{3}$

Lacking a regular determination of the number of HIV-infected women who deliver infants in the United States, the national rate of motherto-child transmission $(2.2 \%)$ was last estimated with the use of the Enhanced Perinatal Surveillance data from the CDC for the period 2005$2008 .{ }^{4}$ The incidence of perinatal HIV-1 infection declined from 4.0 cases per 100,000 live births (in 2008) to 3.1 cases per 100,000 live births (in 2012) but with striking disparities among racial and ethnic groups: the rate was 15.1 cases per 100,000 live births among black infants, 1.7 per 100,000 live births among Hispanic infants, and 0.3 per 100,000 live births among white infants (Fig. 1). ${ }^{5}$ Before and after meeting the targets for the elimination of mother-to-child transmission of HIV-1 for the United States as a whole, we must focus attention and resources on strategies to reduce the high rates of perinatal HIV infection in racial and ethnic minority populations.

Margaret A. Lampe, R.N., M.P.H.

Steven R. Nesheim, M.D.

Eugene McCray, M.D.

Centers for Disease Control and Prevention

Atlanta, GA

mlampe@cdc.gov 